\title{
Macromolecular shape and interactions in layer-by-layer assemblies within cylindrical nanopores
}

\author{
Thomas D. Lazzara ${ }^{1}$, K. H. Aaron Lau ${ }^{2}$, Wolfgang Knoll ${ }^{3}$, Andreas Janshoff ${ }^{4}$ \\ and Claudia Steinem ${ }^{* 1}$
}

\section{Full Research Paper}

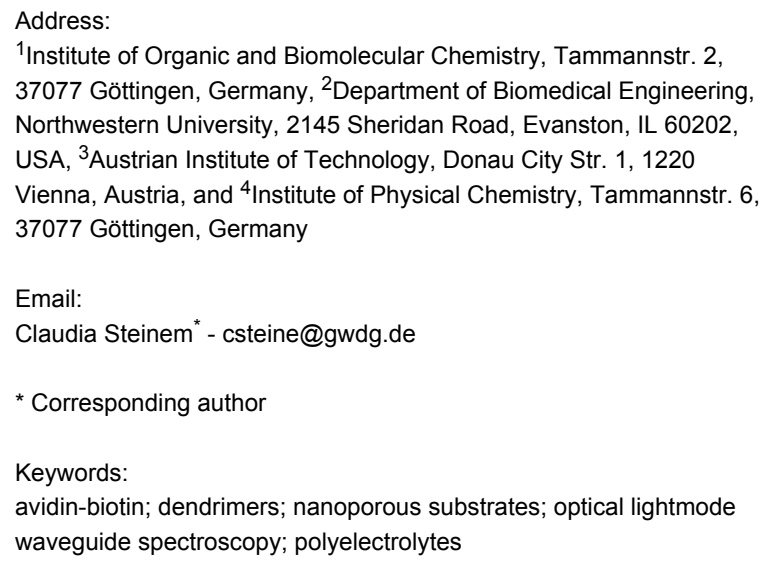

Beilstein J. Nanotechnol. 2012, 3, 475-484. doi:10.3762/bjnano.3.54

Received: 21 February 2012

Accepted: 15 June 2012

Published: 28 June 2012

Associate Editor: J. Rühe

(c) 2012 Lazzara et al; licensee Beilstein-Institut. License and terms: see end of document.

\begin{abstract}
Layer-by-layer (LbL) deposition of polyelectrolytes and proteins within the cylindrical nanopores of anodic aluminum oxide (AAO) membranes was studied by optical waveguide spectroscopy (OWS). AAO has aligned cylindrical, nonintersecting pores with a defined pore diameter $d_{0}$ and functions as a planar optical waveguide so as to monitor, in situ, the LbL process by OWS. The LbL deposition of globular proteins, i.e., avidin and biotinylated bovine serum albumin was compared with that of linear polyelectrolytes (linear-PEs), both species being of similar molecular weight. LbL deposition within the cylindrical AAO geometry for different pore diameters $\left(d_{0}=25-80 \mathrm{~nm}\right)$ for the various macromolecular species, showed that the multilayer film growth was inhibited at different maximum numbers of LbL steps $\left(n_{\max }\right)$. The value of $n_{\max }$ was greatest for linear-PEs, while proteins had a lower value. The cylindrical pore geometry imposes a physical limit to LbL growth such that $n_{\max }$ is strongly dependent on the overall internal structure of the LbL film. For all macromolecular species, deposition was inhibited in native AAO, having pores of $d_{0}=25-30 \mathrm{~nm}$. Both, OWS and scanning electron microscopy showed that LbL growth in larger AAO pores $\left(d_{0}>25-30 \mathrm{~nm}\right)$ became inhibited when approaching a pore diameter of $d_{\text {eff,n_max }}=25-35 \mathrm{~nm}$, a similar size to that of native AAO pores, with $d_{0}=25-30 \mathrm{~nm}$. For a reasonable estimation of $d_{\mathrm{eff}, \mathrm{n} \text { max }}$, the actual volume occupied by a macromolecular assembly must be taken into consideration. The results clearly show that electrostatic LbL allowed for compact macromolecular layers, whereas proteins formed loosely packed multilayers.
\end{abstract}




\section{Introduction}

Layer-by-layer (LbL) deposition is a versatile technique $[1,2]$ to create functional submicrometer thin films and consists of the sequential deposition of functional adsorbing components, to generate multilayered structures. Different functional materials can be stepwise incorporated by LbL, within a single surface structure by electrostatic self-assembly $[3,4]$, molecular-recognition pairs [5-7], or covalent-bond formation [8]. Homogeneous and heterogeneous layered mixing of nanometer-sized species, such as polyelectrolytes, proteins and nanoparticles, has led to various technologically relevant surface coatings [9-13], to the preparation of capsules $[14,15]$ and to functional onedimensional materials, such as nanotubes, by template replication [16-18].

LbL structures on flat surfaces can be well characterized with subnanometer sensitivity by using a number of surface analysis techniques, such as surface plasmon resonance, atomic force microscopy or ellipsometry $[19,20]$. For LbL structures formed inside porous systems, such as within films of colloidal particles or cylindrical nanoporous membranes, the direct investigation of surface processes occurring within nanosized pores has been hampered by the limited availability of in situ, highsensitivity, surface characterization techniques to monitor changes occurring inside the porous morphologies. Techniques such as optical waveguide spectroscopy [21-24] (OWS) and thin-film reflectometry $[25,26]$ have recently been used to independently characterize the thickness and refractive index of optically transparent dielectric thin films.

Here, we studied the formation of $\mathrm{LbL}$ assemblies, obtained by the sequential adsorption of macromolecules within the nanopores of porous anodic aluminum oxide (AAO). The shape and the nature of the interactions between macromolecules were varied. AAO is widely used due to its self-organized, predictable structure, which is composed of nonintersecting, hexagonally close-packed, cylindrical pores running straight through the AAO membrane thickness, with conveniently adjustable monodisperse pore diameters, degree of lattice spacing, and membrane thickness [27-29], making it well suited as a model nanoporous system [30-32].

LbL of polyelectrolyte species within AAO has been experimentally shown to be strongly influenced by $\mathrm{pH}[16]$, ionic strength [23], and steric limitations [23], such that interior deposition can be partially or completely inhibited. On the one hand, electrostatics play a pivotal role in the multilayer growth process involving charged species, in which polyelectrolyte strength, polyelectrolyte chemical structure and solution ionic strength can strongly influence deposition within the nanopores [32]. On the other hand, the confined cylindrical nanopore envi- ronment imposes a steric constraint, in which pore walls are physical barriers that limit the amount of material that can be stepwise incorporated. Confinement in nanoporous environments can, for example, decrease the apparent $\mathrm{p} K_{\mathrm{a}}$ values of cationic polymer brushes, a priori polymerized in pores with $10-40 \mathrm{~nm}$ pore diameters, by more than a full $\mathrm{pH}$ unit [33]. Dobrynin and co-workers have recently simulated the pore-filling behavior during $\mathrm{LbL}$ deposition of both nanoparticle-polyelectrolyte [34] and polyelectrolyte-polyelectrolyte structures [35]. For the fabrication of LbL structures, both steric and electrostatic considerations related to the confined nanoporous geometry generally determine at which point the deposition becomes hindered.

We show here that at the molecular level, parameters such as the macromolecular structure and shape of the LbL building block, as well as the nature of the self-assembly interactions are factors that influence the geometrical arrangement and shape of the growing multilayer film, and therefore modify the point at which hindrance to pore-filling is reached. The LbL deposition of linear polyelectrolytes (linear-PEs) and of globular proteins within AAO nanopores was contrasted to the previously reported behavior of dendrimer polyelectrolytes (dendrimerPEs) [23]. Deposition of these macromolecules in AAO with pore diameters of $d_{0}=63-66 \mathrm{~nm}$, was initially compared with deposition on a planar, charged gold surface. LbL experiments were then carried out in pores with different diameters $d_{0}$, ranging from 25 to $80 \mathrm{~nm}$, until the interior deposition became inhibited. For the cylindrical pore geometry of AAO, the interior deposition was hindered for pore diameters below $30 \mathrm{~nm}$, regardless of the macromolecular structure. In addition, ex situ scanning electron microscopy (SEM) was employed to corroborate the in situ OWS results for linear-PEs.

\section{Results and Discussion}

For our studies on LbL deposition of globular proteins and linear-PE multilayers, we used the nanopores of anodic aluminum oxide (AAO). Scheme 1 (top) shows the general expected internal structure after LbL deposition in AAO nanopores. A two-step anodization process of the AAO ensured highly ordered pores with a low pore-diameter $\left(d_{0}\right)$ size distribution (Scheme 1, bottom). The resulting AAO substrates had an interpore distance of $p=95-105 \mathrm{~nm}$ and a thickness of $h=3.2-3.8 \mu \mathrm{m}$, while the pore diameters were tuned between $d_{0}=25-80 \mathrm{~nm}$ by isotropic pore-widening in phosphoric acid. Before pore diameter adjustment, they were covered with a thin metal coupling layer on the aluminum oxide barrier side (bottom) and then mounted on glass supports by using an optical adhesive [34]. This allowed the characterization of the AAO refractive index and the in situ monitoring of the 


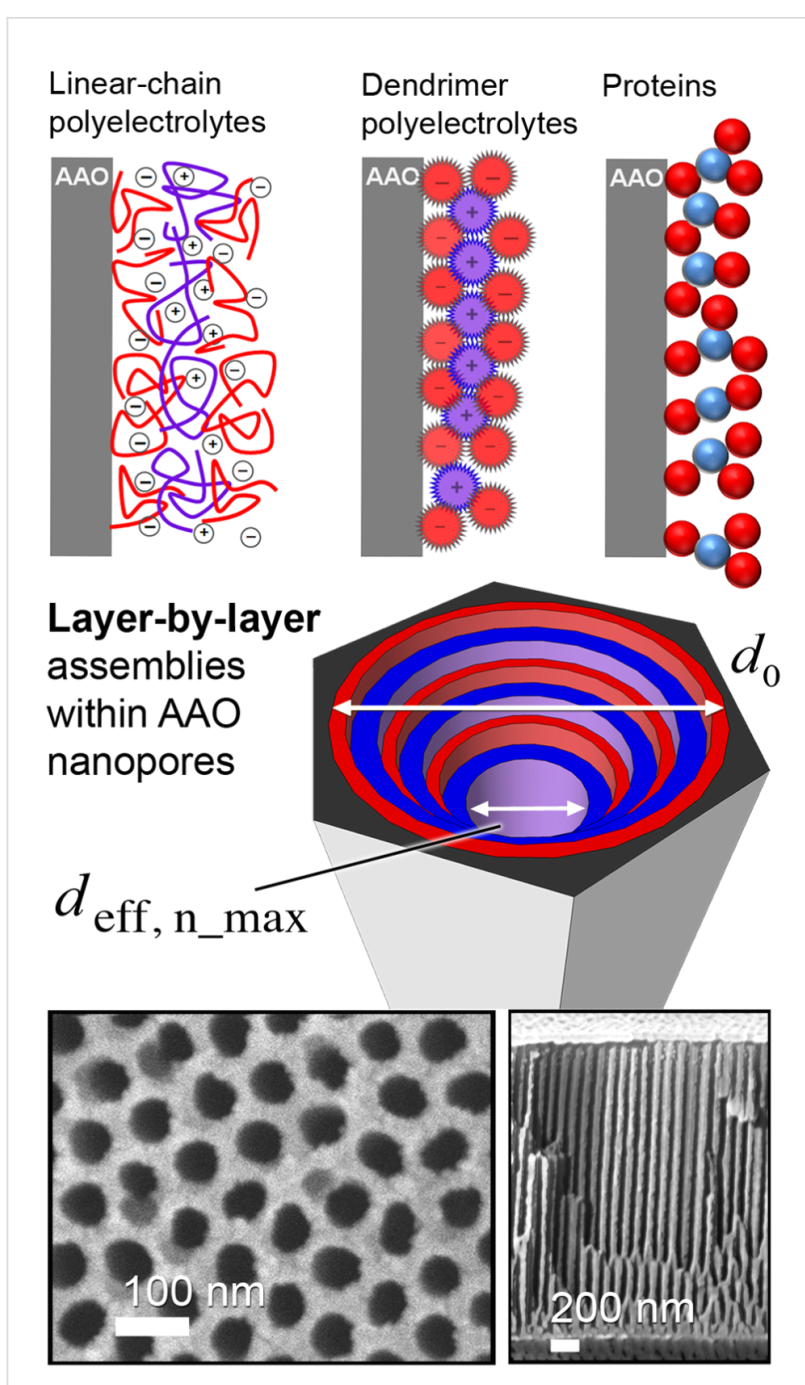

Scheme 1: (Top) Schematic of the layer-by-layer (LbL) structure for different types of macromolecules deposited on the interior AAO pore walls. Polyelectrolyte species (linear chains and dendrimers) adsorb electrostatically, while globular proteins bind by molecular recognition. The LbL process was performed until no further material could be incorporated within the AAO, reaching $d_{\text {eff,n max }}$ (Bottom) SEM of AAO used, shown from the top (left) and in cross-section (right).

macromolecule adsorption kinetics by optical waveguide spectroscopy (OWS). Nanoporosity ensures minimal scattering losses at visible or longer wavelengths, and the Maxwell-Garnett effective medium theory (EMT) can be used to estimate the amount of macromolecular material adsorbed within the AAO nanopores from the experimentally observed changes in the refractive index [22]. This EMT approach relies on the volume fraction contribution of an adlayer on the pore walls, representing an average overall increase in the refractive index of the entire porous material. The approximation is based on the assumption that contiguous layers of uniform thickness are deposited. The film thickness ( $\left.t_{\text {optical }}\right)$ obtained by the optical measurements was estimated assuming that a uniform deposition along the entire length of the pore was achieved. For the porous AAO, $t_{\text {optical }}$ was obtained by fitting the Maxwell-Garnett EMT to the experimentally observed changes of the dielectric constant, providing an average adlayer thickness on the inner pore walls (see Experimental) $[22,23]$.

\section{Layer-by-layer growth}

The influence of the geometric confinement on the LbL process was elucidated by comparing the deposition of different macromolecular species on flat surfaces with that in nanopores. LbL deposition on flat surfaces was measured by surface plasmon resonance (SPR) by using gold substrates with a negatively charged self-assembled monolayer of mercaptohexadecanoic acid. The formation of protein multilayers was achieved by using molecular recognition of biotinylated-bovine serum albumin (b-BSA) by avidin. Avidin has four biotin-binding sites, whereas the b-BSA used has 13 biotin molecules per protein on average. Avidin with a mass of $M_{\mathrm{W}}=66-69 \mathrm{kDa}$, and which is positively charged at neutral $\mathrm{pH}$, was first adsorbed onto the negatively charged surface, followed by b-BSA $\left(M_{\mathrm{W}}=67 \mathrm{kDa}\right)$ adsorption through molecular recognition. Linear-polyelectrolytes (linear-PEs) self-assembled into multilayers by electrostatic interactions between $70 \mathrm{kDa}$ poly(sodium 4-styrene sulfonate) (PSS) and 50-65 kDa poly(allyl amine) hydrochloride (PAH). The positively charged macromolecules were deposited first on the self-assembled mercaptohexadecanoic acid monolayer on gold, followed by the negatively charged linear-PEs. For the porous AAO samples, protein multilayers were grown by first adsorbing avidin electrostatically on the untreated AAO surface, which is negatively charged. For the polyelectrolyte species, the macromolecules were deposited on a positively charged AAO surface obtained by silanization with (3-aminopropyl)-triethoxysilane. In all LbL steps, each adsorption step was continued until the adsorption kinetics showed that saturation was reached. The ionic strength was kept sufficiently high to screen the electrostatic repulsion between same-charge molecules to achieve optimal poreloading conditions.

In Figure 1, the cumulative optical film thickness $t_{\text {optical }}$ obtained for the LbL growth of macromolecules, on a flat surface and within AAO nanopores of $65 \mathrm{~nm}$ diameter, is shown as a function of the number of added layers for both, linear-PEs $\left(\varepsilon_{\text {linear-PEs }}=2.15\right)[36]$ and proteins $\left(\varepsilon_{\text {proteins }}=2.10\right)$ [22]. The estimation of $t_{\text {optical }}$ was made using the same value of dielectric constant for each of the LbL species, in both the planar- and the porous-surface estimates. Comparing the LbL growth on a flat surface versus that within nanopores clearly illustrates how the cylindrical AAO pore geometry imposes a steric limit that terminates the growth of the LbL film after a certain maximum number of deposition steps $\left(n_{\max }\right)$, unlike 

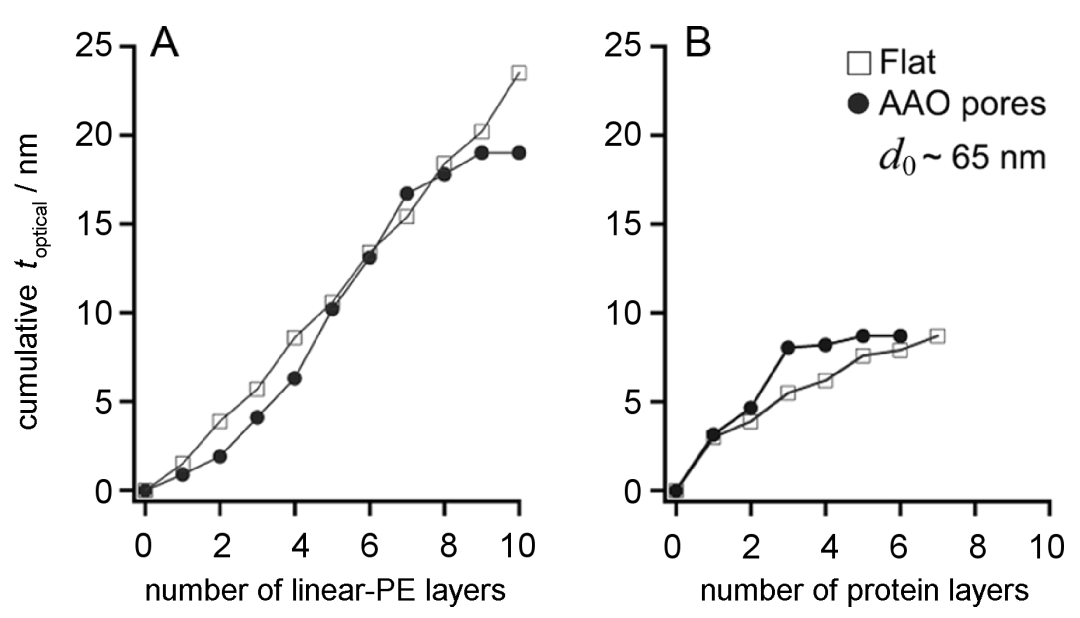

Figure 1: Cumulative optical LbL film thickness ( $\left.t_{\mathrm{optical}}\right)$ for a flat and porous (AAO with $\left.d_{0}=65 \mathrm{~nm}\right)$ substrate, for $(\mathrm{A})$ linear-PEs and $(\mathrm{B})$ proteins. LbL growth becomes hindered in cylindrical nanopores after a certain number of deposited layers.

deposition on a flat surface, which has in principle no steric limit to the number of possible deposition steps. Although the macromolecules discussed here were approximately the same globular size in solution, different $n_{\max }$ were observed for similar pore diameters $d_{0}=65 \mathrm{~nm}$. For linear-PEs $n_{\max }=9$, whereas this value was significantly lower for globular proteins $\left(n_{\max }=3\right)$.

Interestingly, for LbL deposition of dendrimer-polyelectrolytes (Scheme 1) in AAO with pores of the same size, an $n_{\max }=7$ was found (Supporting Information File 1, Figure S1) [23]. These polyelectrolyte dendrimers were N,N-disubstituted hydrazine phosphorus-containing dendrimers of the fourth generation (G4) [37]. Each dendrimer had 96 peripheral charged groups, which were either all cationic or all anionic in nature $\left(\mathrm{G} 4(+)=\mathrm{G}_{4}\left(\mathrm{NH}^{+} \mathrm{Et}_{2} \mathrm{Cl}^{-}\right)_{96}, M_{\mathrm{W}}=32.3 \mathrm{kDa}\right.$; $\mathrm{G} 4(-)=$ $\left.\mathrm{G}_{4}\left(\mathrm{CHCOO}^{-} \mathrm{Na}^{+}\right)_{96}, M_{\mathrm{W}}=36 \mathrm{kDa}\right)$. The mass of these molecules is only half of that of the proteins and linear-PEs, respectively, which would imply that more layers could be deposited in the AAO pores. However, the smaller $n_{\max }$ compared to that obtained for the linear PEs suggests that their structure in the adsorbed state is more globular. This influence of the shape of the adsorbed molecules on $n_{\max }$ was even more clearly observed when contrasting the LbL growth of proteins and linear-PEs over a range of pore diameters $d_{0}=25-80 \mathrm{~nm}$. The cumulative increase in $t_{\text {optical }}$ as a function of the number of added macromolecular layers is shown in Figure 2 for proteins and linear-PEs.
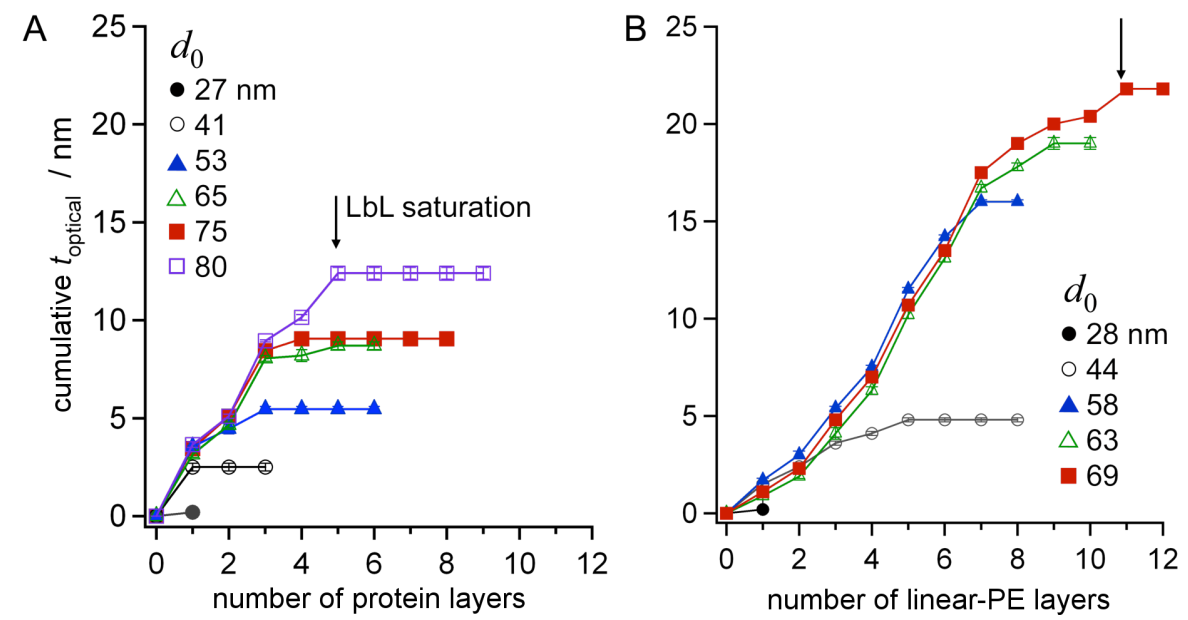

Figure 2: Cumulative optical thickness $t_{\mathrm{optical}}$ of the $\mathrm{LbL}$ multilayer film on the inner surface of the AAO cylindrical nanopores as a function of the number of deposition steps and the AAO pore diameter, for (A) proteins and (B) linear polyelectrolytes. 
The value of $n_{\max }$ increases with larger values of $d_{0}$ for both macromolecules. The striking difference between these two macromolecules is that saturation occurs at significantly lower $n_{\max }$ values for proteins than for linear-PEs, at similar $d_{0}$. For $d_{0}=80 \mathrm{~nm}$, only 5 protein LbL layers could be grown, whereas 11 layers of PSS and PAH were deposited within $d_{0}=69 \mathrm{~nm}$ pores.

The overall multilayer growth process was different for the two molecular species shown in Figure 2. The deposition process of proteins and linear-PEs in cylindrical pores was first characterized by a linear behavior, similar to that for a flat surface (Figure 1). Some deviations were observed for the initial deposition steps for the linear-PEs due to differences in the initial surface charge density, i.e., the number of positively charged silanes on alumina versus negatively charged thiols on gold [38]. Then, for protein multilayers, the LbL deposition saturates rather quickly indicated by $t_{\text {optical }}$, which does not change upon further addition of protein (Figure 2). For linear-PEs, a transition period proceeds for a few deposition steps, characterized by a reduction in $t_{\text {optical }}$ per deposited layer. This reduced deposition is likely due to the onset of hindered diffusion within the nanopore near the pore entrance, which decreases the total amount of material being adsorbed within the porous matrix. Finally, saturation is reached at $n_{\max }$, at which point electrostatic repulsion between same-charge species inhibits the deposition of additional material within the nanopores. The observed behavior, in which the LbL growth in the cylindrical nanopores only proceeded for a certain $n_{\max }$ and terminated before the pore was completely occluded, was also observed for dendrimer-PEs (Supporting Information File 1, Figure S2). Similar experiments involving the formation of polymer nanotubes by LbL of poly(acrylic acid) and $\mathrm{PAH}$, similarly showed that LbL terminates before the pores become completely occluded [16].

In addition to the number of deposited layers, the kinetics of deposition were significantly slower for small pore diameters $\left(d_{0}=25-35 \mathrm{~nm}\right)$ for all types of macromolecules, than they were for larger pores (Supporting Information File 1, Figure S3). The transport of macromolecules within the 3-4 $\mu \mathrm{m}$ long channels was effectively inhibited on the experimental time scales studied ( $<60 \mathrm{~min}$ per deposition step) for pores with diameters of $d_{0}=25-35 \mathrm{~nm}$. (See below for a corresponding scanning electron micrograph of these pores.) In Figure $3, n_{\max }$ is plotted as a function of $d_{0}$, for the globular proteins, linearPEs and dendrimer-PEs. Linear fits to the data show that the slope, i.e., the number of maximum LbL steps, as a function of pore diameter is largest for linear-PEs, while the lowest one was achieved with proteins. The structure of the LbL films therefore influences the effective volume that each macromolecular species occupies. While the mass of the proteins and the linearPEs is very similar, their structure, the nature of the LbL driving force, and the interaction with the AAO surface during adsorption are different. Therefore, the LbL film structure directly influences how much material can be incorporated within the nanopores. For macromolecular species that are deformable, such as the linear-PEs, compact entangled layers are typically formed, while loosely packed layers are expected for rigid, nondeformable species, such as proteins.
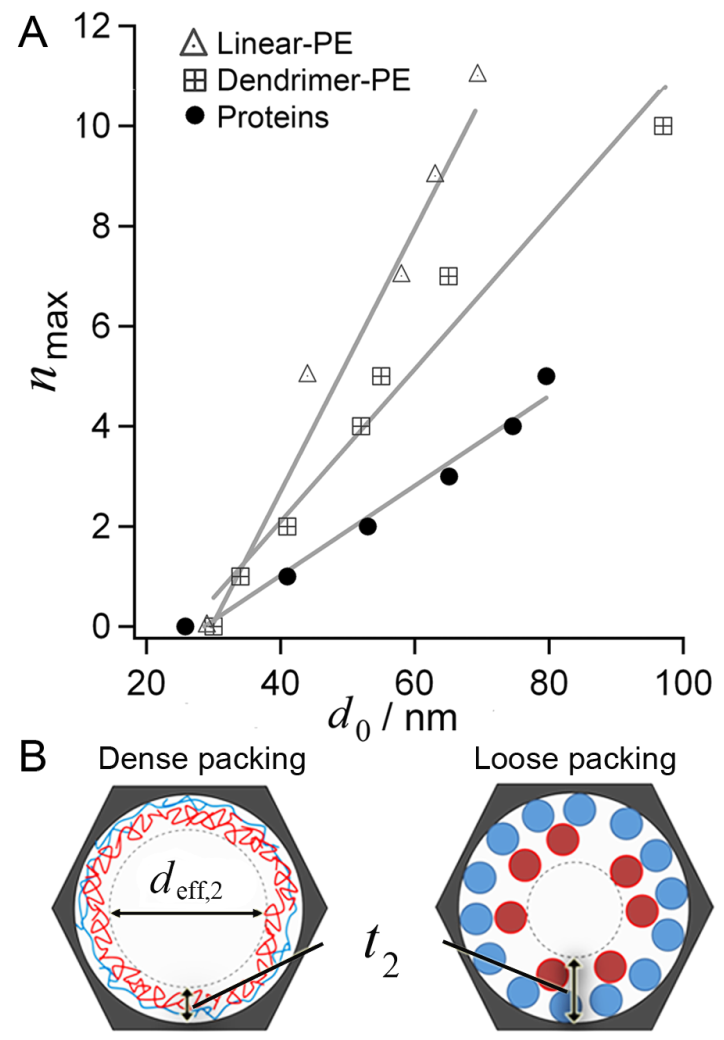

Figure 3: (A) Maximum number of macromolecular LbL steps $\left(n_{\max }\right)$ for proteins, linear-PE and dendrimer-PE multilayers in AAO substrates as a function of initial $d_{0}$. Lines are linear fits to the data. (B) The total volume occupied by a macromolecular layer directly influences the effective film thickness. The effective diameter for two deposited layers $\left(d_{\mathrm{eff}, 2}\right)$ is smaller for proteins since they form loosely packed layers. The LbL film structure determines how much volume is occupied.

In all cases, the volume that the self-assembled film occupies within the pores directly influences the effective pore diameter that remains available $\left(d_{\text {eff }}\right)$ for macromolecular transport within the shrunken pores, after macromolecular adsorption has taken place. This consideration is illustrated in Figure 3B. The value of $d_{\text {eff }}$ is expected to be larger for densely packed flexible linear molecules compared to loosely packed globular ones. 


\section{Macromolecular interactions that limit LbL in nanopores}

Each additional deposited macromolecular layer effectively shrinks the pore diameter that is available for additional macromolecules to travel within the remaining pore. As the number of LbL steps approaches saturation, i.e., $n_{\max }$, the effective pore diameter $\left(d_{\text {eff }}\right)$ reaches a certain value, upon which the cylindrical channel is insufficiently large to allow unhindered diffusion of macromolecules within the pores, a pore diameter referred to as $d_{\text {eff,n_max. }}$. From the experimental data, $d_{\text {eff,n_max }}$ can be calculated as:

$$
d_{\text {eff,n_max }}=d_{0}-2 t_{\text {optical,n_max }}
$$

where $t_{\text {optical,n_max }}$ is the cumulative optical thickness within the pores after LbL growth saturates at $n_{\max }$. The steric hindrance to LbL formation in cylindrical nanopores can be estimated by taking into consideration that macromolecules form adlayers that appear as large as their absolute thickness to incoming macromolecules, regardless of the surface coverage. Therefore, the value of $t_{\text {optical,n_max }}$ in Equation 1 represents a measure of the film thickness that physically limits macromolecular deposition within the pores. For the linear-PEs, $d_{\text {eff,n_max }}$ can be calculated according to Equation 1 to be in the range of 22-34 nm for all initial pore diameters $d_{0}$. The volume occupied by the protein multilayer film, however, is underestimated by the measured cumulative $t_{\text {optical,n_max }}$. This leads to overestimated $d_{\text {eff,n_max }}$ values for the range of $d_{0}$ tested. For all $d_{0}$ values, $d_{\text {eff,n_max }}$ is calculated to be larger than $40 \mathrm{~nm}$. For example, considering an $\mathrm{LbL}$ deposition of proteins in pores with diameters of $d_{0}=80 \mathrm{~nm}, t_{\text {optical,n_max }}=12.5 \mathrm{~nm}$ can be read from Figure $2 \mathrm{~A}$, which results in $d_{\text {eff,n_max }}=55 \mathrm{~nm}$ according to Equation 1. This cannot be correct, since three protein layers $\left(n_{\max }=3\right)$ could be deposited in pores with initial pore diameters of $d_{0}=53 \mathrm{~nm}$ (Figure 2A), and therefore additional depositions would have been possible. Additional considerations are necessary to calculate a correct $d_{\text {eff,n_max }}$, because loosely packed films limit the entrance of molecules to a greater extent than that estimated from the cumulative $t_{\text {optical,n_max. All }}$ of the species studied have similar molecular sizes in solution; however, their interactions with a surface and between the LbL layers differ significantly. Polyelectrolytes can collapse and form dense interpenetrated films, while proteins form looser aggregates due to their shape-persistent nature $[39,40]$. In Figure 3B, we illustrate these differences showing $d_{\text {eff }}$ after the deposition of two layers of either densely or loosely packed macromolecules.

A theoretical calculation of the thickness of each individual layer $t_{\mathrm{calc}, \mathrm{n}}$ should take into account the shape, size and nature of the macromolecular interactions with other macromolecules and with the surface. Ideally, for densely packed layers $t_{\text {optical,n }}$ should equal $t_{\mathrm{calc}, \mathrm{n}}$. For linear-PEs, $t_{\mathrm{calc}, \mathrm{n}}$ was determined to agree with an average value of $t_{\mathrm{optical}, \mathrm{n}}=2.2 \mathrm{~nm}$. However, for loosely packed assemblies $t_{\text {optical,n }}$ is expected to be smaller than $t_{\text {calc,n }}$. A theoretical $d_{\text {eff,n_max }}$ according to:

$$
d_{\text {eff,n_max }}=d_{0}-\sum_{n=0}^{n=n_{\max }} 2 t_{\text {calc, } \mathrm{n}}
$$

can be calculated for the loosely packed protein layers taking the protein dimensions into account. Each protein layer at the pore walls influences the incoming proteins by a reduction in the available cross-sectional area. For avidin, the individual layer thickness $t_{\text {calc, av }}$ was calculated to be $5.3 \mathrm{~nm}$, while for b-BSA $t_{\text {calc,BSA }}$ was calculated to be $6.3 \mathrm{~nm}$. These per-layer thickness values were obtained by taking the average of the three axial protein dimensions, which are $4.0 \times 5.5 \times 6.0 \mathrm{~nm}^{3}$ for avidin and $8.0 \times 8.0 \times 3.0 \mathrm{~nm}^{3}$ for BSA [41-43]. The experimentally determined protein film thicknesses with $t_{\text {optical,av }}=3.2 \mathrm{~nm}$ and $t_{\text {optical,BSA }}=1.1 \mathrm{~nm}$ are indeed considerably smaller than the theoretically calculated ones.

Based on these considerations, $d_{\text {eff,n_max }}$ was calculated for the different macromolecules studied as a function of the initial AAO $d_{0}$ (Figure 4). For linear as well as dendrimer-polyelec-

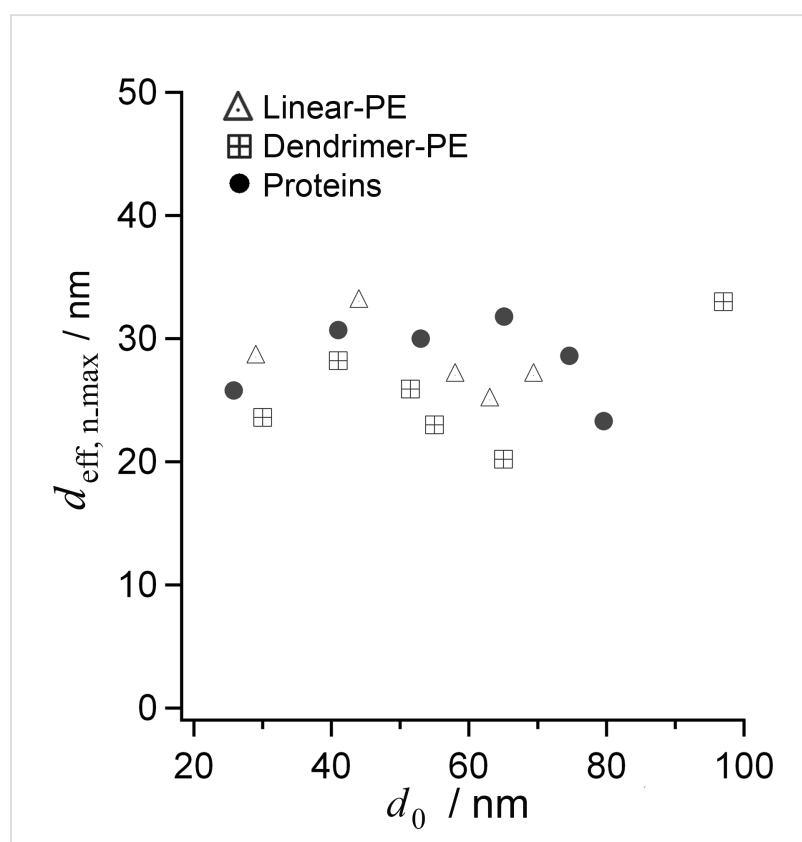

Figure 4: Estimated $d_{\text {eff, } n \text { max }}$ as a function of $d_{0}$, for the studied linear-PEs, dendrimer-PEs and proteins. $d_{\text {eff,n_max }}$ represents the reduced pore diameter when $\mathrm{LbL}$ growth saturates at $n_{\max }$. The results are in agreement with hindered deposition for a native pore diameter of $d_{0}=25-30 \mathrm{~nm}$. 


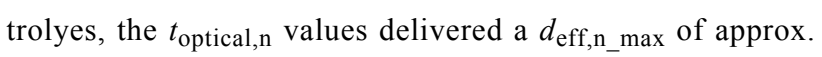
20-35 nm ( $d_{\text {eff,nmax }}=22-34 \mathrm{~nm}$ for linear PEs, and $d_{\text {eff,nmax }}=19-33 \mathrm{~nm}$ for dendrimer-PEs). For proteins, $t_{\text {calc,n_max }}$ gave similar results $\left(d_{\text {eff,n_max }}=21-32 \mathrm{~nm}\right)$ for all $d_{0}$ tested. Taken together, independent of the deposited layer, the minimum effective pore diameter was $d_{\text {eff,n_max }}=20-35 \mathrm{~nm}$ for the three species discussed.

To confirm the approximated minimum effective pore diameter $d_{\text {eff,n_max }}$, we recorded scanning electron microscopy (SEM) images of the substrates. Figure 5 shows SEM images of AAO nanopores with $d_{0}=69 \mathrm{~nm}$ before (A) and after the linear-PE deposition (B), at which point $d_{\text {eff,n_max }}$ was reached, as shown in the corresponding OWS measurement of the reduction of $d_{\mathrm{eff}}$ depicted in Figure 5C. The initial pore diameter $d_{0}$ was reduced to $d_{\text {eff,n_max }}=24 \pm 6 \mathrm{~nm}$, which matches the value obtained by the OWS experiment.

Proteins have internal, defined tertiary structures and therefore tend to deposit more loosely because molecular recognition sites that drive protein-protein interactions are not uniformly available throughout the surface. The proteins studied formed a static system, in which macromolecules effectively became locked into the configuration adopted upon initial binding between proteins, with minimal, if any, further reorganization. The flexibility of polyelectrolytes allows for chain interpenetra- tion $[44,45]$ and surface collapse of the polyelectrolyte structure on charged surfaces [46-48]. Furthermore, polyelectrolyte films are dynamic self-assemblies, in the sense that the internal structure of the film can undergo rearrangements to achieve optimal packing density due to flexible electrostatic interactions.

Furthermore, it is expected that the high degree of dissociation of the charged groups of the linear-PEs used in this study generates LbL multilayer films that are essentially uncharged because of the strong ionic interactions between the polyelectrolyte polymers. The film is basically precipitated onto the surface and forms compact layers. However, LbL films fabricated from weak polyelectrolytes will have a greater tendency to swell in response to ionic strength conditions, $\mathrm{pH}$ and solvent quality. When deposited within cylindrical nanopores, these films may exhibit a behavior intermediate between the proteins and the linear-PEs used. These differences must be considered when the optical thicknesses derived from optical measurements are used to evaluate the point at which macromolecular transport will become hindered during LbL film formation.

\section{Conclusions}

Layer-by-layer (LbL) deposition of different macromolecular species within the cylindrical pores of anodic aluminum oxide (AAO) was strongly dependent on the size and shape of macro-
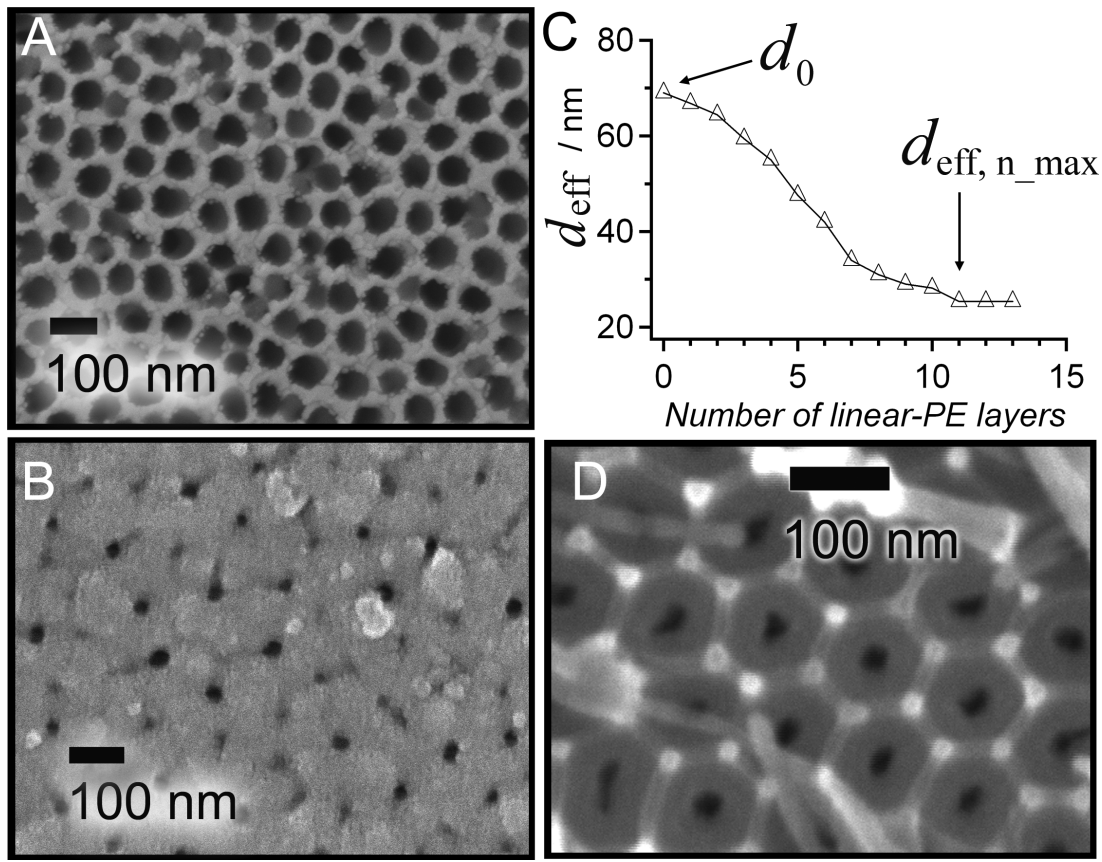

Figure 5: (A) SEM image of AAO with $d_{0}=69 \mathrm{~nm}$ pores before deposition of linear-PEs. (B) SEM image of AAO after 13 PSS and PAH deposition

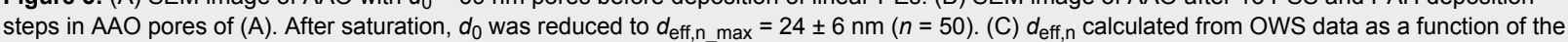
number of linear-PE LbL steps for the sample shown in (A) and (B). (D) SEM of AAO with a pore diameter of $d_{0}=25-30 \mathrm{~nm}$, in which deposition was hindered (see Figure 2). 
molecules, and on the nature of the interactions of these species with the surface and between themselves. The cylindrical pore geometry eventually becomes a physical barrier to LbL growth due to ever increasing confinement after each additional $\mathrm{LbL}$ step. When an effective pore diameter of $20-35 \mathrm{~nm}$ was reached, deposition became inhibited. This was in agreement with hindered deposition of macromolecules within native pores of diameters of $d_{0}=25-30 \mathrm{~nm}$. AAO with different $d_{0}$ were investigated to estimate the average volume that macromolecules occupy for polyelectrolytes, and proteins. The limit at which macromolecular deposition is hindered was not necessarily reflected by simply considering the optical thickness. In fact, the maximum cumulative optical thickness could only be reliably used to calculate the minimum effective pore diameter for polyelectrolytes, because they formed collapsed layers. For proteins, the multilayer LbL film thickness was approximated by using the average protein diameter as an estimate. These results showed that for the cylindrical nanopore geometry, the effective volume occupied by macromolecular species is more relevant to estimate how many LbL steps are possible before the deposition within the pore becomes hindered. In this study, we have only presented experimental results for the formation of homogeneous LbL assemblies, but the steric factors limiting the formation of heterogeneous self-assemblies can be similarly understood. Our results and experimental approach provide insight into tailoring the internal structure of multilayer $\mathrm{LbL}$ assemblies in nanopores towards generating multifunctional LbL films within nanoporous materials.

\section{Experimental Materials}

Lyophilized avidin was purchased from Calbiochem (purity 12.9 units $/ \mathrm{mg}$ ). Biotinylated-bovine serum albumin (b-BSA) with $13 \mathrm{~mol}$ biotin/mol albumin, poly(sodium 4-styrenesulfonate) (PSS) $\left(M_{\mathrm{w}}=70 \mathrm{kDa}\right)$, poly(allylamine hydrochloride) PAH $\left(M_{\mathrm{w}}=50-65 \mathrm{kDa}\right), \mathrm{CuCl}_{2}, \mathrm{NaCl}$, and 16-mercaptohexadecanoic acid (90\%) were purchased from Sigma Aldrich (St. Louis, MO, USA). (3-Aminopropyl)triethoxysilane (APTES) was purchased from Fluka (Steinheim, Germany). Oxalic acid dihydrate was from AppliChem (Darmstadt, Germany) and phosphoric acid 85\% was purchased from Acros Chemicals (New Jersey, NJ, USA). Al foil (0.25 mm thick, purity: 99.999\%) was purchased from Goodfellow (Huntington, UK). High refractive index LaSFN9 glass substrates $(\varepsilon=3.406$ at $632.8 \mathrm{~nm}$ ) were obtained from Hellma Optik (Halle, Germany). The UV-curable optical adhesive (NOA 83H) was purchased from Norland Products (Cranbury, NJ, USA). Ethanol was p.a. grade (VWR, France). The water used was ion exchanged and filtered by using a Millipore system (MilliQ System from Millipore, Molsheim, France; specific resistance $\left.R>18 \mathrm{M} \Omega \mathrm{cm}^{-1}, \mathrm{pH} \sim 5\right)$.

\section{AAO membranes on planar glass supports}

AAO anodized from bulk aluminum foils were mounted on microscope glass slides by using an optical adhesive, according to a previously reported technique [49]. Briefly, AAO membrane thin films were fabricated by electrochemical anodization of aluminum foils after electrochemical polishing. Polished aluminum foils were anodized for $2 \mathrm{~h}$ in $0.3 \mathrm{M}$ oxalic acid, $1{ }^{\circ} \mathrm{C}$ at $40 \mathrm{~V}$. The alumina was removed with $\mathrm{H}_{3} \mathrm{PO}_{4}$ ( 5 vol \%) for $2-3 \mathrm{~h}$. Al foils were then anodized a second time for $1 \mathrm{~h} 35 \mathrm{~min}$ to obtain the desired thickness of $3.5 \mu \mathrm{m}$, or for $2 \mathrm{~h}$ to obtain $5 \mu \mathrm{m}$ thick AAO. Al was removed by an acidic $\mathrm{CuCl}_{2}$ solution until the AAO became visible and no metal remained. Prior to Al removal, the AAO side was isolated from solution by immobilization onto a glass slide and sealed by using epoxy adhesive. The pore diameter of the resulting AAO membranes was widened to the desired diameter by etching in $\mathrm{H}_{3} \mathrm{PO}_{4}(5 \mathrm{vol} \%)$.

\section{Au evaporation}

$\mathrm{Au}$ and $\mathrm{Cr}$ were evaporated on a Bal-Tec MCS610 evaporator equipped with a Bal-Tec QSG100 quartz film-thickness monitor. For the metal layer at the AAO bottom, $2 \mathrm{~nm}$ of $\mathrm{Cr}$ and $25 \mathrm{~nm}$ of Au were evaporated on the AAO barrier layer. For imaging purposes $1 \mathrm{~nm} \mathrm{Cr}$ and $4 \mathrm{~nm} \mathrm{Au}$ were evaporated on SEM samples.

\section{AAO silanization with APTES}

This step was only used with the linear-PEs. For avidin, the protein was adsorbed on the unfunctionalized surface. AAO substrates were $\mathrm{O}_{2}$ plasma cleaned for 2 min immediately prior to gas-phase silanization to increase the surface density of $\mathrm{OH}$ groups. The glass slide substrates to be silanized were inserted into a glass staining jar and $50 \mu \mathrm{L}$ of APTES were added in a glass test tube, inside the chamber. The container was covered with its glass cover and sealed, left in the oven at $130{ }^{\circ} \mathrm{C}$ for 5 min to warm, followed by $3 \mathrm{~h}$ under continuous vacuum.

\section{Surface plasmon resonance (SPR)}

SPR measurements were performed on a setup operating at $632.8 \mathrm{~nm}$ in the Kretschmann configuration [19]. The negatively charged gold surface was obtained by immersion of an $\mathrm{O}_{2}$ plasma cleaned gold surface into a $10 \mathrm{mM}$ mercaptohexadecanoic acid ethanolic solution for $3 \mathrm{~h}$.

\section{Optical waveguide spectroscopy (OWS)}

OWS measurements of the AAO membranes prepared on glass slides were performed on a purpose-built setup [19]. The glassside was attached to the base of a symmetric LaSFN9 glass prism by optical immersion oil $(\varepsilon=2.89)$. The laser $(\lambda=632.8 \mathrm{~nm})$ was incident through the prism-substrate assembly and reflected off the thin metal coupling layer in 
between the AAO and the optical adhesive as the incidence angle $(\theta)$ was varied. At specific $\theta$ values determined by the thickness and the dielectric constant of AAO $\left(\varepsilon_{\mathrm{AAO}}\right)$, the laser was coupled into the AAO film and these waveguide modes were recorded as sharp minima in a reflectivity, $R$, versus $\theta$ scan. Transverse electric (TE) and transverse magnetic (TM) modes were indexed according to the number of nodes in their electromagnetic field distributions. $\varepsilon_{\mathrm{AAO}}$ and the thickness of the AAO film were obtained by fitting the angles of the waveguide mode reflectivity minima using Fresnel simulations carried out with the Winspall program [50]. Tracking the coupling angle of a mode enables real-time, in situ monitoring of changes in the dielectric constant of the film, i.e., adsorption kinetics.

The dielectric constant of AAO $\left(\varepsilon_{\mathrm{AAO}}\right)$ that is measured by OWS includes contributions from the alumina, the pore-filling medium (e.g., buffer), and any organic thin layer coating the pore surfaces (i.e., the LbL multilayer film). The dielectric constant is related to the refractive index by $\varepsilon=n^{2}$. $\varepsilon_{\mathrm{AAO}}$ has anisotropic components that are described by the infinite, prolate ellipsoid approximation within the Maxwell-Garnett theory, and well-described elsewhere [22,51,52]:

$$
\begin{gathered}
\varepsilon_{\mathrm{AAO}}^{\perp}=\varepsilon_{\text {alumina }}+f_{\text {pore }}\left(\varepsilon_{\text {pore }}-\varepsilon_{\text {alumina }}\right) \\
\varepsilon_{\mathrm{AAO}}^{\|}=\varepsilon_{\text {alumina }}+\frac{\varepsilon_{\text {alumina }}+\frac{1}{2}\left(1+f_{\text {pore }}\right)\left(\varepsilon_{\text {pore }}-\varepsilon_{\text {alumina }}\right)}{\varepsilon_{\text {alumina }}+\frac{1}{2}\left(1-f_{\text {pore }}\right)\left(\varepsilon_{\text {pore }}-\varepsilon_{\text {alumina }}\right)}
\end{gathered}
$$

$\varepsilon_{\mathrm{AAO}}^{\perp}$ and $\varepsilon_{\mathrm{AAO}}^{\|}$are, respectively, the dielectric constant components normal and parallel to the AAO membrane surface; $f_{\text {pore }}$ is the pore volume fraction within the AAO, $\varepsilon_{\text {alumina }}=2.68$ is the dielectric constant of bulk anodic alumina at $\lambda=632.8 \mathrm{~nm}$, and $\varepsilon_{\text {pore }}$ is the (effective) dielectric constant within the pores. For a blank AAO film in water, $\varepsilon_{\text {pore }}=$ $\varepsilon_{\text {buffer }}=1.78$. With the addition of an organic film of proteins or linear-PEs $\left(\varepsilon_{\text {proteins }}=2.1, \varepsilon_{\text {linear-PEs }}=2.15\right)$ on the internal pore surfaces, the volume within the pores is occupied by a combination of the organic material and the pore-filling buffer. Recursively applying Equation 3 and Equation 4 for the organic-filled AAO pores, using a new effective $\varepsilon^{\prime}$ pore for the pore interior, provides $\varepsilon_{\mathrm{AAO}}$ after molecular adsorption.

\section{Protein and linear-PE LbL experiments}

Avidin was dissolved in phosphate buffer $\left(20 \mathrm{mM} \mathrm{NaH} \mathrm{PO}_{4} /\right.$ $\mathrm{Na}_{2} \mathrm{HPO}_{4}, 100 \mathrm{mM} \mathrm{NaCl}, \mathrm{pH}=7$ ) to obtain $0.1 \mathrm{mg} / \mathrm{mL}$ solutions. The b-BSA solutions were similarly prepared with $0.1 \mathrm{mg} / \mathrm{mL}$ concentrations. PSS and PAH solutions were prepared with $0.1 \mathrm{mg} / \mathrm{mL}$ concentrations using $500 \mathrm{mM} \mathrm{NaCl}$ in deionized water. For both macromolecules, higher ionic strengths than required were used to significantly reduce the Debye screening length and achieve optimal pore loading. The flow cell was rinsed with ethanol, followed by the buffer. The kinetics were monitored by following the change in a highorder waveguide TM-mode. The solution was passed through the flow cell $\left(15 \times 7.5 \times 0.5 \mathrm{~mm}^{3}\right)$ until 1.4 times the deadvolume had been washed out, and then the solution was recirculated by using a peristaltic pump. The flow rate was kept constant at $0.4 \mathrm{~mL} / \mathrm{min}$.

\section{Supporting Information}

\section{Supporting Information File 1}

Additional figures.

Adsorption kinetics of dendrimer-PEs on flat and porous substrates; adsorption kinetics of avidin and PSS onto AAO with pore diameters of $25-30 \mathrm{~nm}$; cumulative optical thickness of LbL dendrimer multilayer films.

[http://www.beilstein-journals.org/bjnano/content/ supplementary/2190-4286-3-54-S1.pdf]

\section{Acknowledgements}

T. D. L. acknowledges the award of a doctoral scholarship from the Fonds Québécois de Recherche sur la Nature et les Technologies (FQRNT) and additional financial support from the Göttingen Graduate School for Neurosciences and Molecular Biosciences (GGNB).

\section{References}

1. Decher, G. Science 1997, 277, 1232. doi:10.1126/science.277.5330.1232

2. Hammond, P. T. Adv. Mater. 2004, 16, 1271. doi:10.1002/adma.200400760

3. Ali, M.; Yameen, B.; Cervera, J.; Ramirez, P.; Neumann, R.; Ensinger, W.; Knoll, W.; Azzaroni, O. J. Am. Chem. Soc. 2010, 132, 8338. doi:10.1021/ja101014y

4. Lvov, Y.; Ariga, K.; Ichinose, I.; Kunitake, T. J. Am. Chem. Soc. 1995, 117, 6117. doi:10.1021/ja00127a026

5. Ali, M.; Yameen, B.; Neumann, R.; Ensinger, W.; Knoll, W.; Azzaroni, O. J. Am. Chem. Soc. 2008, 130, 16351. doi:10.1021/ja8071258

6. Cassier, T.; Lowack, K.; Decher, G. Supramol. Sci. 1998, 5, 309. doi:10.1016/S0968-5677(98)00024-8

7. Cui, X.; Pei, R.; Wang, Z.; Yang, F.; Ma, Y.; Dong, S.; Yang, X. Biosens. Bioelectron. 2003, 18, 59. doi:10.1016/S0956-5663(02)00114-8

8. Popat, K. C.; Mor, G.; Grimes, C. A.; Desai, T. A. Langmuir 2004, 20, 8035. doi:10.1021/la049075x

9. Bertrand, P.; Jonas, A.; Laschewsky, A.; Legras, R. Macromol. Rapid Commun. 2000, 21, 319. doi:10.1002/(SICI)1521-3927(20000401)21:7<319::AID-MARC319>3.0 .CO;2-7 
10. Crespo-Biel, O.; Dordi, B.; Reinhoudt, D. N.; Huskens, J. J. Am. Chem. Soc. 2005, 127, 7594. doi:10.1021/ja051093t

11. Kotov, N. A.; Dekany, I.; Fendler, J. H. J. Phys. Chem. 1995, 99, 13065. doi:10.1021/j100035a005

12. Tang, Z. Y.; Wang, Y.; Podsiadlo, P.; Kotov, N. A. Adv. Mater. 2006, 18, 3203. doi:10.1002/adma.200600113

13. Yoo, D.; Shiratori, S. S.; Rubner, M. F. Macromolecules 1998, 31 , 4309. doi:10.1021/ma9800360

14. Chen, Y.; Zheng, X.; Qian, H.; Mao, Z.; Ding, D.; Jiang, X. ACS Appl. Mater. Interfaces 2010, 2, 3532. doi:10.1021/am100709d

15. Kim, B.-S.; Lebedeva, O. V.; Kim, D. H.; Caminade, A.-M.; Majoral, J.-P.; Knoll, W.; Vinogradova, O. I. Langmuir 2005, 21, 7200. doi:10.1021/la0504208

16. Cho, Y.; Lee, W.; Jhon, Y. K.; Genzer, J.; Char, K. Small 2010, 6, 2683. doi:10.1002/smll.201001212

17. Hou, S.; Wang, J.; Martin, C. R. Nano Lett. 2005, 5, 231. doi:10.1021/n1048305p

18. Li, J.; Cui, Y. J. Nanosci. Nanotechnol. 2006, 6, 1552. doi:10.1166/jnn.2006.251

19. Knoll, W. Annu. Rev. Phys. Chem. 1998, 49, 569. doi:10.1146/annurev.physchem.49.1.569

20. Porter, M. D.; Bright, T. B.; Allara, D. L.; Chidsey, C. E. D. J. Am. Chem. Soc. 1987, 109, 3559. doi:10.1021/ja00246a011

21. Kovacs, G. J.; Scott, G. D. Appl. Opt. 1978, 17, 3314. doi:10.1364/AO.17.003314

22. Lau, K. H. A.; Tan, L.-S.; Tamada, K.; Sander, M. S.; Knoll, W. J. Phys. Chem. B 2004, 108, 10812. doi:10.1021/jp0498567

23. Lazzara, T. D.; Lau, K. H. A.; Abou-Kandil, A. I.; Caminade, A.-M.; Majoral, J.-P.; Knoll, W. ACS Nano 2010, 4, 3909. doi:10.1021/nn1007594

24. Peic, A.; Staff, D.; Risbridger, T.; Menges, B.; Peter, L. M.; Walker, A. B.; Cameron, P. J. J. Phys. Chem. C 2011, 115, 613. doi:10.1021/jp109316j

25. Alvarez, S. D.; Li, C.-P.; Chiang, C. E.; Schuller, I. K.; Sailor, M. J. ACS Nano 2009, 3, 3301. doi:10.1021/nn900825q

26. Mun, K.-S.; Alvarez, S. D.; Choi, W.-Y.; Sailor, M. J. ACS Nano 2010, 4, 2070. doi:10.1021/nn901312f

27. Li, A. P.; Müller, F.; Gösele, U. Electrochem. Solid-State Lett. 2000, 3, 131. doi:10.1149/1.1390979

28. Li, F.; Zhang, L.; Metzger, R. M. Chem. Mater. 1998, 10, 2470. doi:10.1021/cm980163a

29. Nielsch, K.; Choi, J.; Schwirn, K.; Wehrspohn, R. B.; Gösele, U. Nano Lett. 2002, 2, 677. doi:10.1021/nl025537k

30. O'Sullivan, J. P.; Wood, G. C. Proc. R. Soc. London, Ser. A 1970, 317, 511. doi:10.1098/rspa.1970.0129

31. Thompson, G. E. Thin Solid Films 1997, 297, 192. doi:10.1016/S0040-6090(96)09440-0

32. Thompson, G. E.; Xu, Y.; Skeldon, P.; Shimizu, K.; Han, S. H.; Wood, G. C. Philos. Mag. B 1987, 55, 651. doi:10.1080/13642818708218371

33. Tagliazucchi, M.; Azzaroni, O.; Szleifer, I. J. Am. Chem. Soc. 2010, 132, 12404. doi:10.1021/ja104152g

34. Carrillo, J.-M. Y.; Dobrynin, A. V. ACS Nano 2011, 5, 3010. doi:10.1021/nn200065q

35. Carrillo, J.-M. Y.; Dobrynin, A. V. Langmuir 2011, 28, 1531. doi:10.1021/la203940w

36. Feldötö, Z.; Varga, I.; Blomberg, E. Langmuir 2010, 26, 17048. doi:10.1021/la102351f

37. Caminade, A.-M.; Majoral, J.-P. Acc. Chem. Res. 2004, 37, 341. doi:10.1021/ar020077n
38. Niemeyer, C. M.; Boldt, L.; Ceyhan, B.; Blohm, D. Anal. Biochem. 1999, 268, 54. doi:10.1006/abio.1998.3017

39. Edmiston, P. L.; Saavedra, S. S. J. Am. Chem. Soc. 1998, 120, 1665. doi:10.1021/ja972634k

40. Ram, M. K.; Bertoncello, P.; Ding, H.; Paddeu, S.; Nicolini, C. Biosens. Bioelectron. 2001, 16, 849. doi:10.1016/S0956-5663(01)00208-1

41. Klajnert, B.; Stanisławska, L.; Bryszewska, M.; Pałecz, B. Biochim. Biophys. Acta, Proteins Proteomics 2003, 1648, 115. doi:10.1016/S1570-9639(03)00117-1

42. Livnah, O.; Bayer, E. A.; Wilchek, M.; Sussman, J. L. Proc. Natl. Acad. Sci. U. S. A. 1993, 90, 5076. doi:10.1073/pnas.90.11.5076

43. Pugliese, L.; Coda, A.; Malcovati, M.; Bolognesi, M. J. Mol. Biol. 1993, 231, 698. doi:10.1006/jmbi.1993.1321

44. Liu, G.; Zhao, J.; Sun, Q.; Zhang, G. J. Phys. Chem. B 2008, 112, 3333. doi:10.1021/jp710600f

45. Schmitt, J.; Gruenewald, T.; Decher, G.; Pershan, P. S.; Kjaer, K.; Loesche, M. Macromolecules 1993, 26, 7058. doi:10.1021/ma00077a052

46. Castelnovo, M.; Joanny, J.-F. Langmuir 2000, 16, 7524. doi:10.1021/la000211h

47. Dobrynin, A. V.; Rubinstein, M. Prog. Polym. Sci. 2005, 30, 1049. doi:10.1016/j.progpolymsci.2005.07.006

48. Xie, A. F.; Granick, S. Macromolecules 2002, 35, 1805. doi:10.1021/ma011293z

49. Lazzara, T. D.; Lau, K. H. A.; Knoll, W. J. Nanosci. Nanotechnol. 2010, 10, 4293. doi:10.1166/jnn.2010.2189

50. Scheller, A. WinSpall, Version 3.01; Max-Planck Institute for Polymer Research: Mainz, Gernany; Reflectivity simulation program solving Fresnel Equations.

51. Kim, D. H.; Lau, K. H. A.; Joo, W.; Peng, J.; Jeong, U.; Hawker, C. J.; Kim, J. K.; Russell, T. P.; Knoll, W. J. Phys. Chem. B 2006, 110, 15381. doi:10.1021/jp0630469

52. Kim, D. H.; Lau, K. H. A.; Robertson, J. W. F.; Lee, O.-J.; Jeong, U.; Lee, J. I.; Hawker, C. J.; Russell, T. P.; Kim, J. K.; Knoll, W. Adv. Mater. 2005, 17, 2442. doi:10.1002/adma.200500170

\section{License and Terms}

This is an Open Access article under the terms of the Creative Commons Attribution License (http://creativecommons.org/licenses/by/2.0), which permits unrestricted use, distribution, and reproduction in any medium, provided the original work is properly cited.

The license is subject to the Beilstein Journal of Nanotechnology terms and conditions: (http://www.beilstein-journals.org/bjnano)

The definitive version of this article is the electronic one which can be found at: doi: $10.3762 /$ bjnano.3.54 